\title{
Prophylactic Probiotics for Preterm Infants: A Systematic Review and Meta-Analysis of Observational Studies
}

\author{
Rie Olsen ${ }^{a}$ Gorm Greisen ${ }^{b}$ Morten Schrøder ${ }^{b}$ Jesper Brok ${ }^{c, d}$ \\ ${ }^{a}$ Faculty of Health and Medical Sciences, University of Copenhagen, and Departments of ${ }^{b}$ Neonatology and \\ ${ }^{c}$ Pediatrics, Copenhagen University Hospital, Rigshospitalet, Copenhagen, Denmark; ${ }^{d}$ Cancer Section, University \\ College London Institute of Child Health, London, UK
}

\section{Key Words}

Probiotics · Preterm neonates - Necrotizing enterocolitis .

Mortality · Meta-analysis · Observational studies

\begin{abstract}
Background: Necrotizing enterocolitis (NEC) is a major morbidity and cause of mortality in preterm neonates. Probiotics seem to have a beneficial role in preventing NEC, which is confirmed in meta-analyses of randomized controlled trials (RCTs). We therefore aimed to review and confirm the efficacy of probiotics in preterm neonates obtained in observational studies. Objective: To assess the effects of prophylactic probiotics in preterm infants. Methods: A meta-analysis was performed searching PubMed, EMBASE, CENTRAL (the Cochrane Library) and www.clinicaltrials.gov. Reference lists of reviews of RCTs were also searched. Included studies were observational studies that enrolled preterm infants $<37$ weeks of gestational age. Trials were included if they administered any probiotics and measured at least one clinical outcome (e.g. NEC, all-cause mortality, sepsis or long-term development scores). Two authors extracted characteristics and outcomes from included studies. The Newcastle-Ottawa Scale was used for quality assessment. A random-effects meta-analysis mod-
\end{abstract}

el was used, and heterogeneity was assessed by the $\mathrm{I}^{2}$ test. Results: We included 12 studies with 10,800 premature neonates $(5,144$ receiving prophylactic probiotics and 5,656 controls). The meta-analysis showed a significantly decreased incidence of NEC (risk ratio, RR $=0.55,95 \%$ confidence interval, $95 \% \mathrm{Cl}, 0.39-0.78 ; \mathrm{p}=0.0006)$ and mortality $(\mathrm{RR}=0.72,95 \%$ $\mathrm{Cl}, 0.61-0.85 ; \mathrm{p}<0.0001)$. Sepsis did not differ significantly between the two groups $(\mathrm{RR}=0.86,95 \% \mathrm{Cl}, 0.74-1.00 ; \mathrm{p}=$ 0.05). Conclusions: Probiotic supplementation reduces the risk of NEC and mortality in preterm infants. The effect sizes are similar to findings in meta-analyses of RCTs. However, the optimal strain, dose and timing need further investigation.

(c) 2015 S. Karger AG, Basel

\section{Introduction}

Necrotizing enterocolitis (NEC) is the most common gastrointestinal emergency in neonates, primarily affecting premature neonates [1]. Following advances in neonatology and modern neonatal intensive care units the incidence of NEC has increased, with improved survival of smaller, more premature infants [2]. However, despite a great amount of research several issues are still hamper-

\section{KARGER}

E-Mail karger@karger.com

www.karger.com/neo
(C) 2015 S. Karger AG, Basel

$1661-7800 / 15 / 1092-0105 \$ 39.50 / 0$
Rie Olsen

Faculty of Health and Medical Sciences

University of Copenhagen

Blegdamsvej 9, DK-2100 Copenhagen (Denmark)

E-Mail rieolsen91@gmail.com 
ing our understanding of this disease, and NEC continues to be a major cause of death in preterm infants [3].

It has been suggested that pathogenesis must be multifactorial and may involve an overactive response of the immune system to cause an insult that might be ischemic, infectious, related to the introduction of enteric feeds or a response to translocation of normal enteric bacteria [4]. This overactive inflammatory response may lead to harmful effects. Accordingly, there is a critical role for both medical and surgical management in the treatment of NEC once it occurs, but prevention could have the most dramatic impact on overall morbidity [4]. Such a preventive measure could be the enteral administration of probiotics.

The term probiotics is defined as 'live microorganisms which when administered in adequate amounts confer a benefit for the host' [5]. The beneficial mechanisms of probiotics are unknown but include changes in intestinal permeability, enhanced mucosal IgA responses and increased production of anti-inflammatory cytokines [1]. The immature immune system of premature neonates cannot control the outgrowth of pathogenic bacteria. According to the benefits of probiotics, feeding premature infants with these bacteria may populate their intestines with normal flora and prevent an overgrowth of pathogenic flora that contribute to the development of NEC [6].

Several systematic reviews of randomized controlled trials (RCTs) on prophylactic probiotics for preterm infants have found beneficial effects on NEC and mortality $[1,7]$. Despite such favorable results, concerns about the effectiveness of prophylactic probiotics still remain. Hence, we wanted to explore any possible beneficial effects of probiotics in observational studies beyond the effects previously observed in RCTs. We therefore aimed to carry out a systematic review on observational (nonrandomized) studies to evaluate the efficacy of probiotic supplementation outside strictly controlled settings.

\section{Methods}

We primarily followed guidelines from the Cochrane Handbook of Systematic Reviews of Interventions [8] and the MOOSE guidelines for meta-analysis and systematic reviews of observational studies, supplemented by PRISMA guidelines [9]. The study protocol was registered in the PROSPERO database for systematic reviews (reg. No. CRD42014014464).

\section{Eligibility Criteria}

Quantitative nonrandomized studies comparing prophylactic probiotics to a standard regime for preterm infants were eligible for inclusion. Studies had to include at least 20 participants (ges- tational age $<37$ weeks or birth weight $<2,500 \mathrm{~g}$ ). All probiotic regimes were included, as well as combinations (e.g. Lactobacillus acidophilus and Bifidobacterium lactis), and the intervention had to be administered for at least 7 days. Twins were also included, but neonates with significant birth defects were excluded (e.g. severe heart disease, myelomeningocele).

The primary outcome of interest was NEC at stage II or III according to Bell's criteria [10] and, if not clearly described, any NEC stage as defined by the individual clinical trial. Other outcome measures included all-cause mortality, sepsis or any long-term neurological development.

\section{Data Sources and Search Strategies}

We searched PubMed, EMBASE and CENTRAL (the Cochrane Central Register of Controlled Trials, The Cochrane Library) in February 2015 with the search terms 'premature infant' or 'preterm neonate' or 'prematurity' and 'probiotic' or 'lactobacillus' or 'saccharomyces' or 'bifidobacterium' or 'streptococcus' and 'necrotizing enterocolitis'. The full strategy of the PubMed search is available in Appendix. Language was restricted to English, Danish, Norwegian or Swedish. If only the abstract was in English, the study was included. Reference lists of identified studies and systematic reviews of RCTs were also searched. In addition, we searched for relevant ongoing trials at www.clinicaltrials. gov.

R.O. and J.B. searched the literature independently and assessed the eligibility of trials for inclusion in the review. Disagreements were settled by discussion and if necessary a third author (G.G.) was consulted. The same authors as listed above performed the study extraction, and disagreements were settled similarly by discussion until consensus was reached. The following data were extracted from each study: author, date/year of publication, country, type of observational study, age of infants, duration of intervention (including age at the first day of intervention), dosage, type of probiotic and outcomes: NEC, all-cause mortality, sepsis and any long-term neurological development.

\section{Quality Assessment of Included Studies}

The quality of included trials was assessed by R.O. and J.B. using the Newcastle-Ottawa Scale (NOS) [11], which was modified to fit our study design: 0-3 stars indicate poor study quality, 4-6 stars indicate acceptable study quality, and 7-9 stars indicate good study quality. In the event of disagreements, consensus was reached by discussion.

\section{Statistical Analysis}

For the meta-analysis, forest plots were calculated using weighted scores and a random-effects model. We chose the random-effects model over the fixed-effects model because it accounts for variations between studies, which we anticipated due to the inclusion of different types of intervention, different cohort designs and different ways to assess outcomes. The degree of heterogeneity across studies was determined with the $\mathrm{I}^{2}$ test with $\mathrm{I}^{2}$ values of $25 \%$ or less, $50 \%$ and $75 \%$ or greater representing low, moderate and high inconsistency [12]. p $<0.05$ was considered significant and $95 \%$ confidence intervals (95\% CI) were calculated. Funnel plots assessed the risk of bias, including publications bias. All statistical calculations were done with the assistance of Review Manager (version 5.2.11). 
Fig. 1. Flow chart of selection of studies included in the meta-analysis.

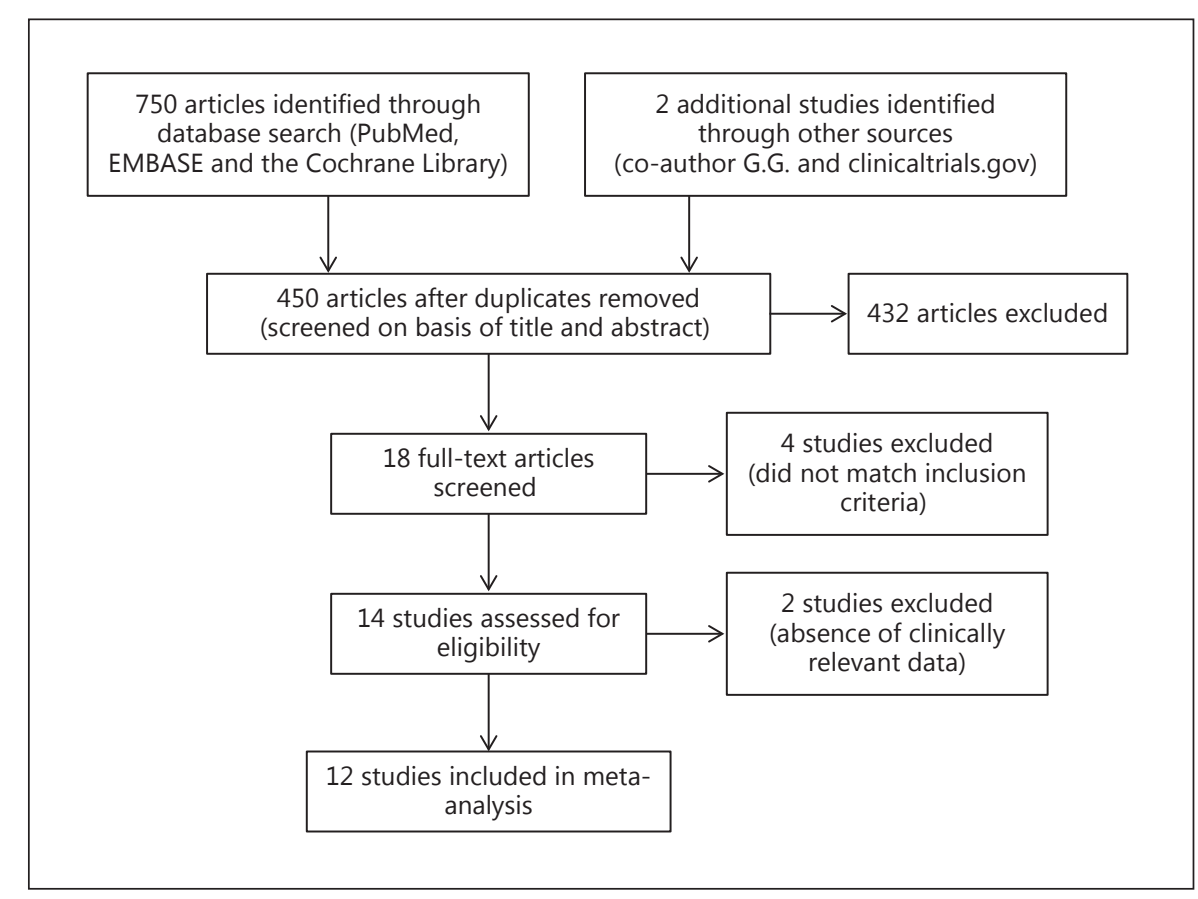

\section{Results}

\section{Description of Trials}

We identified 752 articles (fig. 1). After the removal of duplications and reviewing abstracts or original publications we found 14 studies that met the eligibility criteria. Two studies $[13,14]$ had insufficient data presentation (only on preterm neonates) and were therefore not included in the meta-analysis calculation. Hence, 12 studies were pooled in the meta-analysis, including a total of 10,800 neonates [15-26]. Details of the selection process are shown in figure 1.

The main characteristics of the included studies are summarized in table 1, including the quality assessment of the included studies. Studies had moderate-to-good quality according to NOS (except 1 study for which only the abstract was available). The included studies covered a large geographical area. All included studies were longitudinal cohort studies, either retrospective $[15,16,19$, $22,23]$, prospective $[17,18,20,21,24,26]$ or with a historical control group [25]. Ten of the included studies were single-center studies and 2 were multicenter [17, 23]. With the exception of 2 studies [16, 18], all studies classified NEC according to Bell's criteria $\geq$ stage II [10].

\section{Effects of Intervention}

All trials included in the meta-analysis reported data on definite NEC (fig. 2a). In the group treated with prophylactic probiotics a smaller proportion of preterm neonates developed NEC $(169 / 5,144,3.3 \%)$ compared to the control group $(325 / 5,656,5.7 \%)$. The use of prophylactic probiotics was associated with a significant decreased risk of NEC (risk ratio, RR $=0.55,95 \% \mathrm{CI}, 0.39-0.78 ; \mathrm{p}=$ $0.0006)$ compared to the control group. We observed significant heterogeneity between the studies $\left(\mathrm{I}^{2}=58 \%\right.$; $\mathrm{p}=$ $0.006)$. To assess the publication bias in this meta-analysis, we made a funnel plot (fig. 3). Visual interpretation of this funnel plot showed some asymmetry, which could indicate bias, e.g. publication bias.

Data for mortality was available in 9 trials (fig. 2b). In the probiotic group the mortality rate was $7.6 \%(354 / 4,629)$ compared to the control group with $10 \%(353 / 3,510)$. This demonstrated a significant reduction in the risk of mortality $(\mathrm{RR}=0.72,95 \% \mathrm{CI}, 0.61-0.85 ; \mathrm{p}<0.0001)$ with low heterogeneity between the studies $\left(I^{2}=10 \% ; \mathrm{p}=0.35\right)$. The funnel plot was symmetric, indicating no bias.

Data for sepsis was available in 7 trials (fig. 2c). A metaanalysis showed no statistically significant reduced risk in the group given prophylactic probiotics $(\mathrm{RR}=0.86,95 \%$ CI, 0.74-1.00; $\mathrm{p}=0.05$ ) compared to the control group. We observed moderate heterogeneity between the studies $\left(\mathrm{I}^{2}=36 \% ; \mathrm{p}=0.15\right)$, and the funnel plot was symmetric. 
Table 1. Characteristics of included trials

\begin{tabular}{|c|c|c|c|c|c|c|}
\hline Study & $\begin{array}{l}\text { Neonates on } \\
\text { probiotics, } n\end{array}$ & $\begin{array}{l}\text { Controls, } \\
\mathrm{n}\end{array}$ & $\begin{array}{l}\text { Birth weight or } \\
\text { gestational age }\end{array}$ & Probiotic agent & $\begin{array}{l}\text { Primary } \\
\text { outcome }\end{array}$ & $\begin{array}{l}\text { NOS } \\
\text { score }\end{array}$ \\
\hline $\begin{array}{l}\text { Bonsante [15], } 2013 \\
\text { France }\end{array}$ & 347 & 783 & $>24$ and $<31$ weeks & L. casei rhamnosus & $\begin{array}{l}\text { NEC; sepsis; } \\
\text { mortality }\end{array}$ & 7 \\
\hline $\begin{array}{l}\text { Dang [16], } 2015 \\
\text { USA }\end{array}$ & 128 & 135 & $\begin{array}{l}<1,250 \mathrm{~g} \text { and } / \\
\text { or }<28 \text { weeks }\end{array}$ & $\begin{array}{l}\text { L. rhamnosus GG/ } \\
\text { B. infantis }\end{array}$ & NEC; mortality & 7 \\
\hline $\begin{array}{l}\text { Hoyos }[18]^{\mathrm{a}}, 1999 \\
\text { Colombia }\end{array}$ & 102 & 103 & $<1,500 \mathrm{~g}$ & L. acidophilus/B. infantis & NEC; mortality & 8 \\
\hline $\begin{array}{l}\text { Hunter [19], } 2012 \\
\text { USA }\end{array}$ & 79 & 232 & $<1,500 \mathrm{~g}$ & L. reuteri & NEC; sepsis & 7 \\
\hline $\begin{array}{l}\mathrm{Li}[22], 2013 \\
\text { California }\end{array}$ & 291 & 289 & $<1,500 \mathrm{~g}$ & $\begin{array}{l}\text { Mixture of Streptococcus and } \\
\text { Bifidobacterium }^{c}\end{array}$ & NEC; mortality & 7 \\
\hline $\begin{array}{l}\text { Luoto [23], } 2010 \\
\text { Finland }\end{array}$ & 418 & 1,900 & $\begin{array}{l}<30 \text { weeks } \\
\text { or }<1,500 \mathrm{~g}\end{array}$ & Lactobacillus GG & NEC & 8 \\
\hline $\begin{array}{l}\text { Repa [24], } 2014 \\
\text { Austria }\end{array}$ & 230 & 233 & $<34$ weeks & L. acidophilus/B. infantis & $\begin{array}{l}\text { NEC; sepsis; } \\
\text { mortality }\end{array}$ & 6 \\
\hline $\begin{array}{l}\text { Yamashiro [25], } 2010 \\
\text { Japan }\end{array}$ & 338 & 226 & $<1,500 \mathrm{~g}$ & B. breve & $\begin{array}{l}\text { NEC; sepsis; } \\
\text { mortality }\end{array}$ & 6 \\
\hline
\end{tabular}

a Data for $<1,500$ g obtained by contacting the authors. ${ }^{\mathrm{b}}$ Mixture of Bifidobacterium (breve, bifidum, infantis and longum) and L. rhamnosus. ${ }^{\mathrm{c}}$ Mixture of S. thermophilus, B. infantis and B. bifidum.

\section{Discussion}

In this meta-analysis of observational studies, we found that treating premature infants with prophylactic probiotics was associated with a statistically significant decreased incidence of NEC and mortality. These findings support results found in meta-analyses of RCTs, which have been frequently debated $[7,27]$.

\section{Strengths and Limitations}

One of the strengths of the current study is that this review includes an exhaustive and reproducible search strategy according to a registered protocol. Another strength is the relatively large number of preterm infants $(\mathrm{n}=10,800)$ included in the meta-analysis. The included studies covered a geographically large area, thereby making the results applicable to a mixed population of premature infants. In addition, the current review included several studies published within the last few years, thus providing an up-to-date assessment of prevention of NEC in preterm neonates treated with prophylactic probiotics. Finally, the average study quality of the included studies was rated as acceptable on the NOS, and until now there exists no official threshold for distinguishing between poor, acceptable and good quality studies.

However, there are several limitations to our analysis. One limitation is that all included studies were observational studies ranking in the lower end of the evidence hierarchy with increased risk of, for example, selection bias. Therefore, this meta-analysis of observational stud- 
a Effects of probiotics on NEC

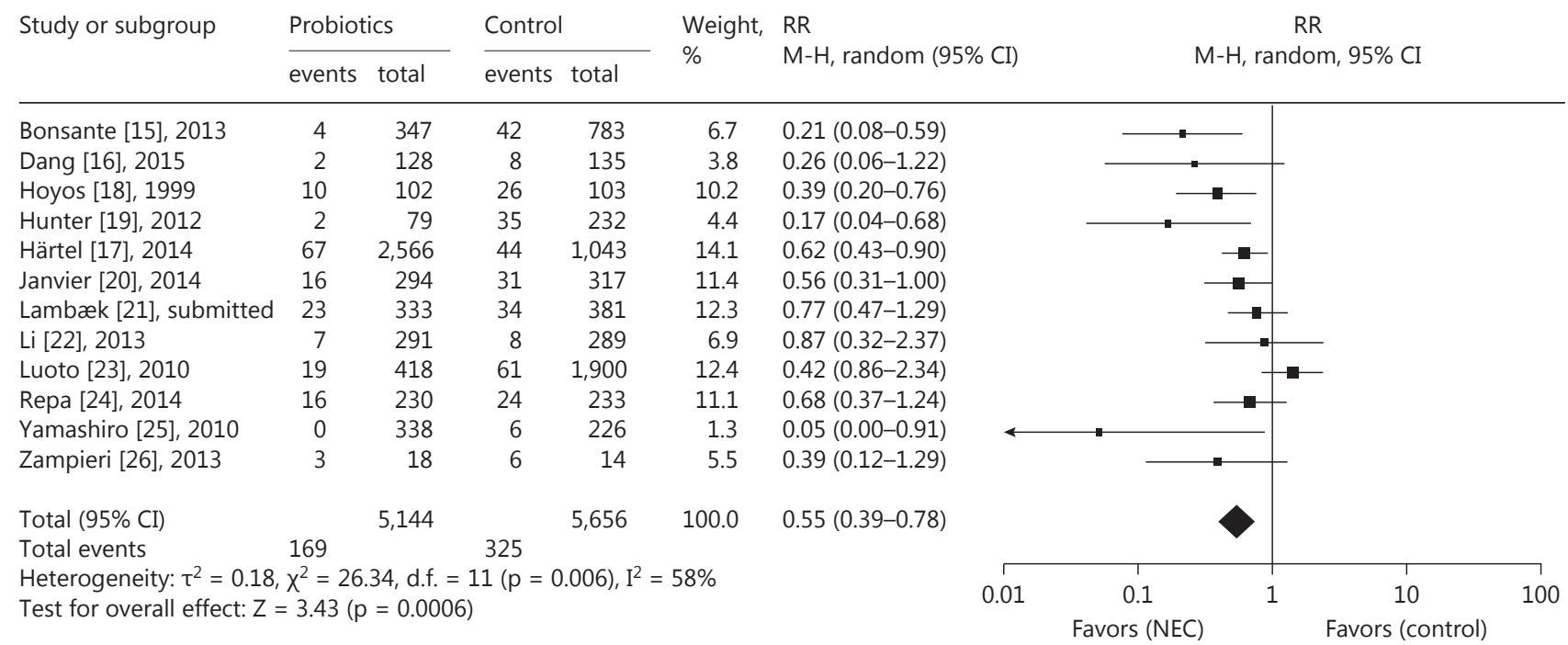

b Effects of probiotics on mortality

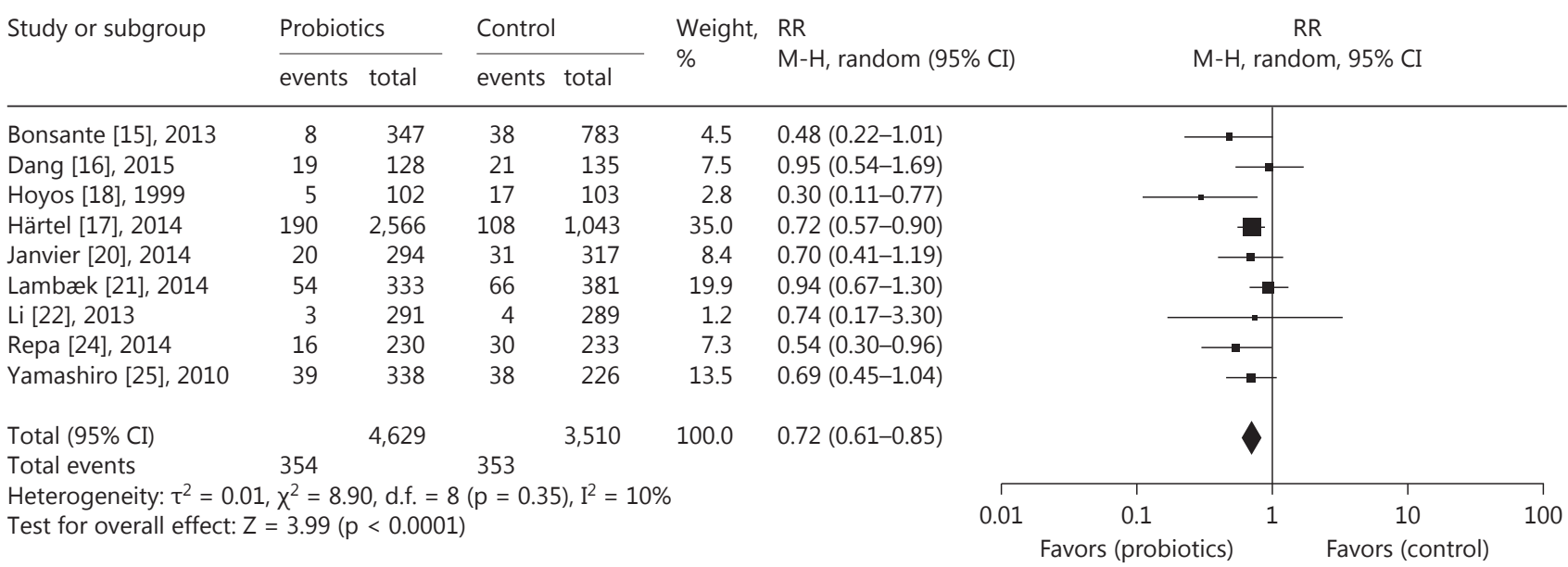

C Effects of probiotics on sepsis

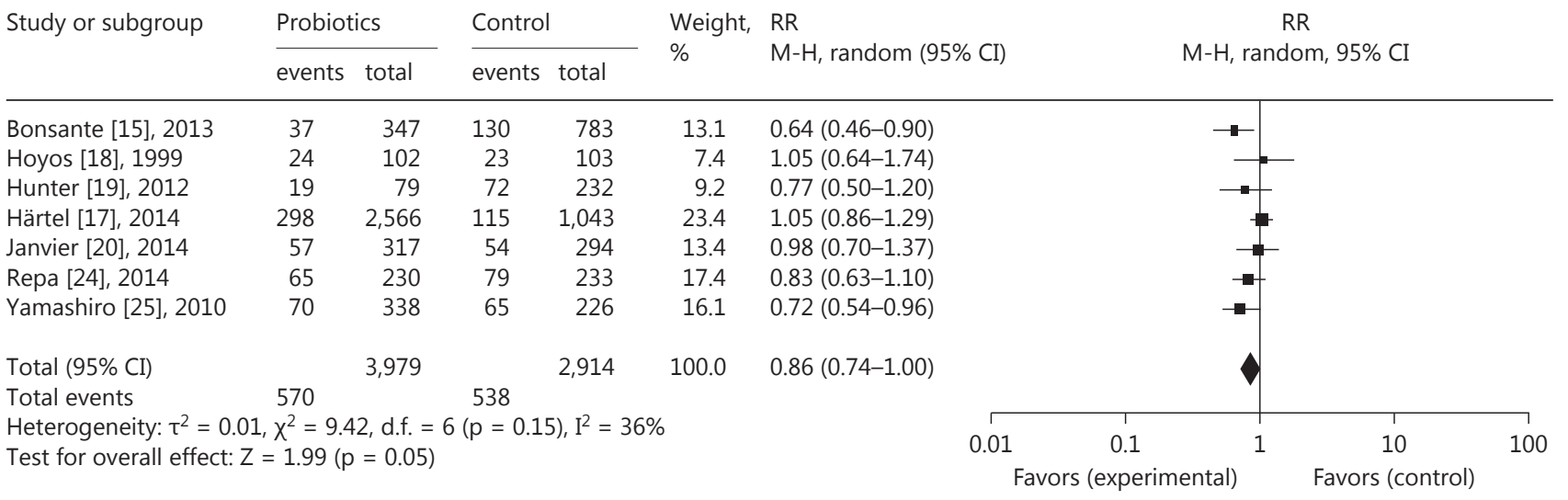

Fig. 2. Forest plot of prophylactic probiotics for preterm neonates. $\mathrm{M}-\mathrm{H}=$ Mantel-Haenszel method.

Prophylactic Probiotics for Preterm Infants
Neonatology 2016;109:105-112 DOI: $10.1159 / 000441274$ 


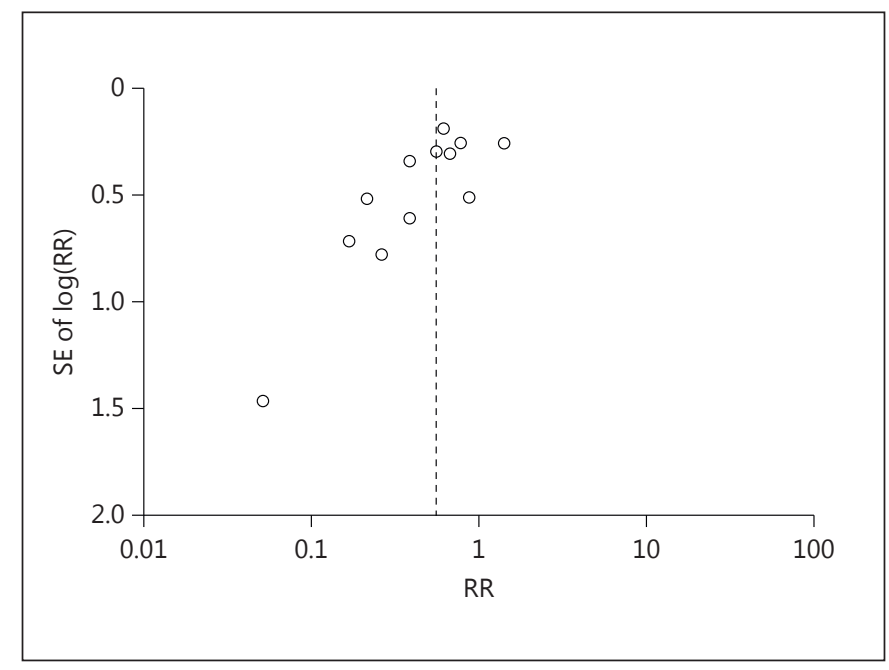

Fig. 3. Funnel plot (NEC).

ies provides much weaker evidence than meta-analyses of RCTs. However, although several meta-analyses of RCTs have already been conducted, to our knowledge this is the first meta-analysis of non-RCTs, and it was relevant to assess whether the experimental data from the meta-analyses of RCTs could be 'confirmed' with data from a daily clinical setting. Another limitation is that the overall estimate of the meta-analysis should be interpreted with caution since the degree of heterogeneity was relatively high for NEC $\left(\mathrm{I}^{2}=58 \%\right)$. Such between-study heterogeneity could be caused by differences in eligibility criteria in each study and the use of different types of probiotic agents, dosage and timing (e.g. when the probiotic supplement was first started). In addition, the included studies had different policies towards enteral feeds (e.g. breast milk vs. formula) and the use of antibiotics. Almost all of the included neonates were delivered by cesarean section in one of the studies [25]; this may also have affected our results since delivery by cesarean section has been shown to consistently delay the establishment of a stable and functionally active neonatal intestinal flora [28]. We included all studies on preterm infants (gestational age $<37$ weeks), but the infants in the publications in this metaanalysis in general were very preterm (gestational age $<32$ weeks). Since the risk of NEC is in inverse ratio with weight and gestational age, this may have affected our results. The funnel plot (NEC) indicated publication bias towards more positive results for NEC but not for mortality or sepsis. In the NEC meta-analysis one of the included studies was unpublished [21].
Interestingly, our results support reviews of RCTs, which reported that probiotic supplements decrease the incidence of NEC and mortality in preterm infants [7, 27]. The effect sizes and CIs are also very similar, with an almost 50\% reduction in NEC and one third reduction in mortality. Similarly, we observed a trend towards a benefit in the reduction of sepsis; however, this was not statistically significant.

Besides the 12 studies included in the meta-analysis we identified 2 observational studies, which included both term and preterm neonates. We were unable to extract the data on premature neonates, which is why these studies were not included in the meta-analysis calculation. However, the combined group showed a reduced incidence of NEC and mortality. One of these studies [13] included 197 term and preterm neonates with birth weights $>1,000 \mathrm{~g}$, and in this study NEC was less frequent in the probiotic group $(0 / 78,0 \%)$ compared to the control group $(6 / 119,5 \% ; p=0.044)$. The mortality rate was also significantly lower; in the probiotic group the mortality rate was $2.6 \%(2 / 78)$ compared to the control group with $10.1 \%(12 / 119 ; \mathrm{p}=0.037)$. The other study [14] that included a total of 2,528 neonates showed a significant decrease of NEC in the probiotic group $(6 / 1,182,0.51 \%)$ compared to the control group $(19 / 1,346,1.41 \%$; p < $0.05)$.

Unfortunately, no trials presented adequate data on long-term neurological development. Therefore, this area needs further investigation, as previous studies suggest that NEC might lead to long-term neurodevelopmental impairment [29].

One of the included studies [16] provided data on the subgroup of extremely premature infants (gestational age $<28$ weeks). This study showed no statistically significant decrease of NEC and mortality. No other studies provided data on this subgroup of neonates. Therefore, the use of probiotics in extremely premature infants needs further investigation, especially considering the limited data from RCT studies [7].

\section{Perspective}

Over the past years RCTs and meta-analyses of RCTs have indicated beneficial effects of probiotics. Our metaanalysis now supports this finding as it shows positive effects of probiotic supplementation also outside strict controlled settings.

While there is evidence of the benefits of probiotics, there are still concerns about strain, dose and timing of administration. In a retrospective analysis Schmolzer et al. [30] found a statistically significant decreased risk of 
NEC in the group receiving early NEC prophylaxis (e.g. antibiotics, probiotics). This shows a tendency towards the benefits of early treatment, but this needs further investigation. Deshpande et al. [31] composed a guideline for the use of probiotics in preterm neonates based on the evidence available and concluded that a combination of Lactobacillus and Bifidobacterium is preferred. The dose should be at least $3 \times 10^{9}$ organisms per day, starting when the neonate is ready for enteral feeds, and continued until 35 weeks' corrected age or discharge. However, there are few RCTs comparing different regimens head-to-head, and there is little evidence for choosing any specific type or protocol. Therefore, it is being widely discussed whether it is time to change practice or wait until more evidence has shown beneficial effects [32-34]. However, based on the results we present here and on the results from metaanalyses of RCTs, combined with the cost of probiotics, it seems relevant to consider this intervention.

\section{Conclusion}

For the first time in a meta-analysis of non-RCTs we have confirmed the results from meta-analyses of RCTs showing that prophylactic probiotic supplementation can reduce the risk of NEC and mortality in preterm infants. However, there is still little evidence for choosing any particular type of probiotic or any specific protocol administration.

\section{Appendix}

\section{PubMed}

(()(()((()(()(()("bifidobacterium"[MeSH $\quad$ Terms] OR "bifidobacterium"[All Fields]) OR b.[All Fields]) AND ("sulfalene"[MeSH Terms] OR "sulfalene"[All Fields] OR "longum"[All Fields])) OR "saccharomyces boulardii"[All Fields]) OR ("Streptococcus thermophilus"[Mesh] OR "Streptococcus thermophilus"[All Fields])) OR ((("bifidobacterium"[MeSH
Terms] OR "bifidobacterium"[All Fields]) OR b.[All Fields]) AND longus[All Fields])) OR (("bifidobacterium"[MeSH Terms] OR "bifidobacterium"[All Fields]) OR b.[All Fields]) AND lactis[All Fields])) OR (("'bifidobacterium"[MeSH Terms] OR "bifidobacterium"[All Fields]) OR b.[All Fields]) AND breve[All Fields])) OR (("'bifidobacterium"[MeSH Terms] OR "bifidobacterium"[All Fields]) OR b.[All Fields]) AND bifidus[All Fields])) OR((("lactobacillus"[MeSHTerms] OR"lactobacillus"[All Fields]) OR L.[All Fields]) AND bulgaricus[All Fields])) OR ("Lactobacillus reuteri"[Mesh] OR ((("lactobacillus"[MeSH Terms] OR "lactobacillus"[All Fields]) OR L.[All Fields]) AND reuteri[All Fields])))OR((("lactobacillus"[MeSHTerms] OR"lactobacillus"[All Fields]) OR L.[All Fields]) AND sporogenes[All Fields])) OR ("Lactobacillus plantarum"[Mesh] OR (("lactobacillus"[MeSH Terms] OR "lactobacillus"[All Fields]) OR L.[All Fields]) AND plantarum[All Fields]))) OR ("Lactobacillus rhamnosus"[Mesh] OR (("lactobacillus"[MeSH Terms] OR "lactobacillus"[All Fields]) OR L.[All Fields]) AND (rhamnosus[All Fields] OR GG[All Fields])))) OR $\quad(((($ lactobacillus"[MeSH $\quad$ Terms $] \quad$ OR "lactobacillus"[All Fields]) OR L.[All Fields]) AND casei[All Fields]) OR "Lactobacillus casei"[Mesh])) OR ("Lactobacillus acidophilus"[Mesh] OR ((("lactobacillus"[MeSH Terms] OR "lactobacillus"[All Fields]) OR L.[All Fields]) AND acidophilus[All Fields]))) OR ("Probiotics"[Mesh] OR (("probiotics"[MeSH Terms] OR "probiotics"[All Fields] OR "probiotic"[All Fields]) OR ("probiotics"[MeSH Terms] OR "probiotics"[All Fields])))) AND ("Infant, Premature"[Mesh] OR ((premature[All Fields] OR preterm[All Fields] OR neonatal[All Fields] OR ("infant, newborn"[MeSH Terms] OR ("infant"[All Fields] AND "newborn"[All Fields]) OR "newborn infant"[All Fields] OR "neonate"[All Fields])) AND (("infant"[MeSH Terms] OR "infant"[All Fields]) OR ("infant"[MeSH Terms] OR "infant"[All Fields] OR "infants"[All Fields]) OR ("infant, premature"[MeSH Terms] OR ("infant"[All Fields] AND "premature"[All Fields]) OR "premature infant"[All Fields] OR "prematurity"[All Fields]) OR ("infant, newborn"[MeSH Terms] OR ("infant"[All Fields] AND "newborn"[All Fields]) OR "newborn infant"[All Fields] OR "neonate"[AllFields]))))) AND("Enterocolitis,Necrotizing"[Mesh] OR ((Necrotising[All Fields] OR necrotizing[All Fields]) AND ("enterocolitis"[MeSH Terms] OR "enterocolitis"[All Fields]))).

\section{Acknowledgment}

The authors wish to thank Professor of Communication, Kim C. Schrøder.

\section{References}

Prophylactic Probiotics for Preterm

Infants
1 Deshpande G, Rao S, Patole S: Probiotics for prevention of necrotising enterocolitis in preterm neonates with very low birthweight: a systematic review of randomised controlled trials. Lancet 2007;369:1614-1620.

2 Henry MCW, Moss RL: Necrotizing enterocolitis. Annu Rev Med 2009;60:111-124.

3 Neu J: Necrotizing enterocolitis: the mystery goes on. Neonatology 2014;106:289-295.

4 Berman L, Moss RL: Necrotizing enterocolitis: an update. Semin Fetal Neonatal Med 2011; $16: 145-150$
5 Joint FAO/WHO Working Group Report on Drafting Guidelines for the Evaluation of Probiotics in Food. London, FAO/WHO, 2002.

6 Soll RF: Probiotics: are we ready for routine use? Pediatrics 2010;125:1071-1072.

7 Alfaleh K, Anabrees J: Probiotics for prevention of necrotizing enterocolitis in preterm infants. Cochrane Database Syst Rev 2014; 4:CD005496.

8 Higgins JPT, Sally Green S (eds): Cochrane Handbook for Systematic Reviews of Interventions. Chichester, Wiley, 2008. 
9 Liberati A, Altman DG, Tetzlaff J, Mulrow C, Gøtzsche PC, Ioannidis JPA, et al: The PRISMA statement for reporting systematic reviews and meta-analyses of studies that evaluate health care interventions: explanation and elaboration. J Clin Epidemiol 2009;62:e1-e34.

10 Bell MJ, Ternberg JL, Feigin RD, Keating JP, Marshall R, Barton L, et al: Neonatal necrotizing enterocolitis. Therapeutic decisions based upon clinical staging. Ann Surg 1978;187:1.

11 Wells G, Shea B, O'Connel D, Peterson J, Welch V, Losos M, et al: The Newcastle-Ottawa Scale (NOS) for Assessing the Quality of Nonrandomised Studies in Meta-Analyses 2013. Ottawa, University of Ottawa, 2013.

12 Higgins JP, Thompson SG, Deeks JJ, Altman DG: Measuring inconsistency in meta-analyses. BMJ 2003;327:557-560.

13 Ozen M, Elmas A, Ormeci AR, Dinleyici EC: Prophylactic probiotic supplementation not only decreases the incidence of NEC and mortality but also nosocomial infections in neonates in a cohort study. Int Symp Probiotics Prebiotics Pediatr, Istanbul, 2012, p 91.

14 Li L, Wu B, Gao X, Wang S, Zheng Z, Xu J: Role of probiotics in the prevention of neonatal necrotizing enterocolitis: a case-control study (in Chinese). Chin J Contemp Pediatr 2006;8:464-466.

15 Bonsante F, Iacobelli S, Gouyon J-B: Routine probiotic use in very preterm infants: retrospective comparison of two cohorts. Am J Perinatol 2013;30:41-46.

16 Dang S, Shook L, Garlitz K, Hanna M, Desai $\mathrm{N}$ : Nutritional outcomes with implementation of probiotics in preterm infants. J Perinatol 2015;35:447-450.

17 Härtel C, Pagel J, Rupp J, Bendiks M, Guthmann F, Rieger-Fackeldey E, et al: Prophylactic use of Lactobacillus acidophilus/Bifidobacterium infantis probiotics and outcome in very low birth weight infants. J Pediatr 2014; 165:285-289.e1.
18 Hoyos AB: Reduced incidence of necrotizing enterocolitis associated with enteral administration of Lactobacillus acidophilus and Bifidobacterium infantis to neonates in an intensive care unit. Int J Infect Dis 1999;3:197-202.

19 Hunter C, Dimaguila MAVT, Gal P, Wimmer JE, Ransom JL, Carlos RQ, et al: Effect of routine probiotic, Lactobacillus reuteri DSM 17938, use on rates of necrotizing enterocolitis in neonates with birthweight $<1,000$ grams: a sequential analysis. BMC Pediatr 2012;12: 142.

20 Janvier A, Malo J, Barrington KJ: Cohort study of probiotics in a North American neonatal intensive care unit. J Pediatr 2014;164: 980-985.

21 Lambæk I, Fonnest G, Gormsen M, Greisen G: Routine probiotic prophylaxis for necrotising enterocolitis in very preterm infants. A planned historically controlled study. Dan Med J, submitted.

22 Li D, Rosito G, Slagle T: Probiotics for the prevention of necrotizing enterocolitis in neonates: an 8-year retrospective cohort study. J Clin Pharm Ther 2013;38:445-449.

23 Luoto R, Matomäki J, Isolauri E, Lehtonen L: Incidence of necrotizing enterocolitis in verylow-birth-weight infants related to the use of Lactobacillus GG. Acta Paediatr 2010;99: $1135-1138$.

24 Repa A, Thanhaeuser M, Endress D, Weber M, Kreissl A, Binder C, et al: Probiotics (Lactobacillus acidophilus and Bifidobacterium bifidum) prevent NEC in VLBW infants fed breast milk but not formula. Pediatr Res 2015; 77:381-388.

25 Yamashiro Y, Nagata S: Beneficial microbes for premature infants, and children with malignancy undergoing chemotherapy. Benef Microbes 2010;357-365.
26 Zampieri N, Pietrobelli A, Biban P, Soffiati M, Dall'agnola A, Camoglio FS: Lactobacillus paracasei subsp. paracasei F19 in Bell's stage 2 of necrotizing enterocolitis. Minerva Pediatr 2013;65:353-360.

27 Wang Q, Dong J, Zhu Y: Probiotic supplement reduces risk of necrotizing enterocolitis and mortality in preterm very low-birthweight infants: an updated meta-analysis of 20 randomized, controlled trials. J Pediatr Surg 2012;241-248.

28 Fanaro S, Chierici R, Guerrini P, Vigi V: Intestinal microflora in early infancy: composition and development. J Paediatr 2003;48-55.

29 Schulzke SM, Deshpande GC, Patole SK: Neurodevelopmental outcomes of very lowbirth-weight infants with necrotizing enterocolitis: a systematic review of observational studies. Arch Pediatr Adolesc Med 2007;161: 583-590.

30 Schmolzer G, Urlesberger B, Haim M, Kutschera J, Pichler G, Ritschl E, et al: Multimodal approach to prophylaxis of necrotizing enterocolitis: clinical report and review of literature. Pediatr Surg Int 2006;573-580.

31 Deshpande GC, Rao SC, Keil AD, Patole SK: Evidence-based guidelines for use of probiotics in preterm neonates. BMC Med 2011;9:92.

32 Ofek Shlomai N, Deshpande G, Rao S, Patole S: Probiotics for preterm neonates: what will it take to change clinical practice? Neonatology 2013;64-70.

33 Modi N: Probiotics and necrotising enterocolitis: the devil (as always) is in the detail. Commentary on N Ofek Shlomai et al: Probiotics for preterm neonates: what will it take to change clinical practice? (Neonatology 2014; 105:64-70). Neonatology 2014;105:71-73.

34 Jacobs SE, Tobin JM, Opie GF, Donath S, Tabrizi SN, Pirotta M, et al: Probiotic effects on late-onset sepsis in very preterm infants: a randomized controlled trial. Pediatrics 2013; 132:1055-1062. 